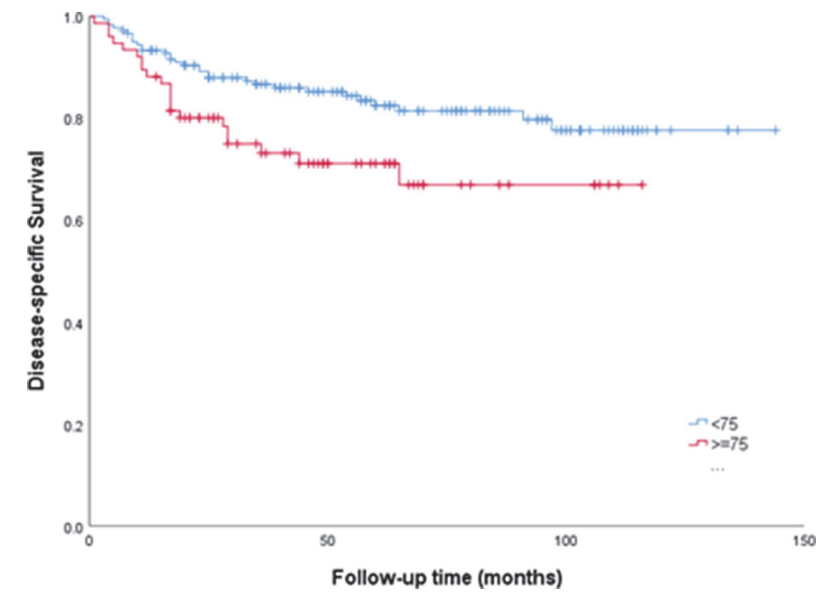

Abstract 632 Figure 1

Result(s)* In total 283 patients were included, 185 were classified as NOP while 98 as OP. No differences were found in disease characteristics. Laparoscopy was performed in $82 \%$ of patients. Both pelvic and para-aortic lymphadenectomy were performed more frequently in NOP than in OP $(56,8 \%$ vs. $36,7 \% ; \mathrm{p}<0,01$ and 45,9 vs. $22,9 \% ; \mathrm{p}<0,01)$. Rates of intra-operative $(6,5 \%$ vs $12,4 \%, \mathrm{p}=0,12)$ and post-operative $(13,5 \%$ vs $20,6 \% \quad p=0,13)$ complications were similar between NOP and OP respectively, as well as the severity of complications according to Clavien-Dindo classification $(4,4 \%$ vs $7,4 \%$ grade III, $0,5 \%$ vs $0 \%$ grade IV, $\mathrm{p}=0,51)$. No differences were found in other surgical and post-operative variables. The 5-year disease-specific survival (DSS) rate was lower in OP $(66,9 \%$ vs $86,0 \%, p=0,02)$. When analyzing only patients who underwent complete staging surgery (107 of the NOP and 35 of the OP), no differences were found in perioperative complications rate. In this subgroup, there were no differences in DSS between NOP and OP $(78,1 \%$ vs $71,0 \%, \mathrm{p}=0,64)$.

Conclusion* OP do not present a higher rate of perioperative complications compared to NOP. However, they underwent less lymphadenectomies and presented poorer DSS. Considering only patients in whom complete surgery was performed, OP presented similar DSS to NOP, without presenting a higher rate of perioperative complications.

\section{ENDOCRINE THERAPY IN ADVANCED ENDOMETRIOID ENDOMETRIAL CANCER: A RETROSPECTIVE ANALYSIS OF CLINICOPATHOLOGIC FACTORS}

${ }^{1} \mathrm{M}$ Ray*, ${ }^{1} \mathrm{R}$ Kim, ${ }^{1} \mathrm{~J}$ Mirkovic, ${ }^{1} \mathrm{~N}$ Dhani, ${ }^{1} \mathrm{E}$ Donovan, 'E Leung, ${ }^{2} \mathrm{M}$ Ennis, ${ }^{1} \mathrm{H}$ Mackay, ${ }^{1} \mathrm{~K}$ Jerzak. ${ }^{1}$ Toronto, Toronto, Canada; ${ }^{2}$ Applied Statistician, Markham, Canada

\subsection{6/ijgc-2021-ESGO.178}

Introduction/Background* Endocrine therapy (ET) is a well-tolerated treatment strategy among women with low grade, hormone receptor positive advanced endometrioid endometrial cancer (EC).

Methodology In this retrospective cohort study, we identified patients with advanced endometrioid EC who were treated with ET between 2016-2018 by a medical oncologist at the Sunnybrook Odette Cancer Centre (Toronto, Canada). Descriptive analyses were performed. Median PFS from the time of starting ET and OS from diagnosis of advanced disease were assessed using Kaplan Meier methods. Predictors of PFS were evaluated using Cox regression models.

Result(s)* Twenty nine patients were included. Median age at diagnosis of advanced disease was 65.7 years. The majority of patients had grade $1(55 \%)$ or grade $2(29 \%)$ EC. Twenty three patients $(79 \%)$ had ER and/or PR positive tumors $(\geq 1 \%$ using immunohistochemistry); ER/PR status was negative in 1 case and unknown for 5 patients. Only $17 \%$ of patients received chemotherapy for advanced disease prior to starting ET. Letrozole (52\%) and progestins (48\%) were the most frequently used. Interestingly, the majority of patients (79\%) received radiotherapy for oligoprogression while receiving ET.

Median PFS was 12.8 months. Median OS has not been reached, however, $73 \%$ of patients survived at least 4 years [95\% Confidence Interval (CI) $56.4 \%$ to $95.5 \%$ ]. Use of a progestin as first-line ET was associated with a longer PFS [Hazard Ratio (HR) 0.42; 95\%CI 0.18-0.97, p=0.04], with a trend toward longer OS [HR 0.20 ; 95\%CI $0.04-1.06$, $\mathrm{p}=0.06]$. Lack of oligoprogression requiring radiotherapy was associated with a longer PFS [HR 0.23; 95\%CI 0.07-0.83, $\mathrm{p}=0.02]$, but not OS. Patient age, tumor grade, time to diagnosis of metastatic disease, stage at initial diagnosis, and use of chemotherapy prior to ET were not significantly associated with PFS or OS.

Conclusion* The clinical benefit of ET was greater in our cohort compared to prior published reports, possibly due to selection of patients with low grade and ER/PR positive tumors. The use of first-line progestins and lack of oligoprogression requiring radiotherapy were significantly associated with longer PFS in this small cohort.

\section{SENTINEL LYMPH NODE IN ENDOMETRIAL CANCER: OUR EXPERIENCE IN THE UNIVERSITY HOSPITAL 12 DE OCTUBRE IN MADRID}

${ }^{1} G$ Lopez Gonzalez*, 'MDLR Oliver, ${ }^{2} R$ Benabdallah, ${ }^{1} M$ Ortega Bravo, ${ }^{3} \mathrm{TM}$ José, ${ }^{4} \mathrm{~L}$ Parrilla-Rubio, ${ }^{1} \mathrm{JM}$ Seoane-Ruiz, ${ }^{1} \mathrm{C}$ Alvarez, ${ }^{1} \mathrm{~B}$ Gil Ibanez, ${ }^{1} \mathrm{~A}$ Tejerizo. ${ }^{1}$ Hospital Universitario 12 de Octubre, Gynecology and obstetrics, Madrid, Spain; ${ }^{2}$ Hospital Universitario 12 de Octubre, Medicine School, Madrid, Spain; ${ }^{3}$ Hospital Universitario 12 de Octubre, Nuclear Medicine, Madrid, Spain; ${ }^{4}$ Hospital Universitario 12 de Octubre, Anatomic Pathology, Madrid, Spain

\subsection{6/ijgc-2021-ESG0.179}

Introduction/Background* The goal of this study is to review the sentinel lymph node (SLN) in endometrial cancer in the University Hospital 12 de Octubre in Madrid from June 2016 to October 2020. The aim is to know the demographic and clinical features of the patients and to assess the outcomes of SLN in our population with technetium99 (Tc99), indocyanine green (ICG) or blue dye.

Methodology

Result(s)* From June 2016 to October 2020, 166 patients diagnosed with endometrial cancer underwent surgery in our hospital. In $34.4 \%(n=57)$ of them SLN was performed and included in this review.

Demographic and clinical features are shown in table 1. $80.7 \%(\mathrm{n}=46)$ were classified as low risk endometrial cancer and $19,3 \%(n=11)$ as intermediate risk.

Laparoscopy was the most frequent approach (96.4\%). Median operative time was 203 minutes (IQR, 173 to 249).

A combined tracer technique was used in $75.4 \%$ cases. Most of them combining Tc99 and ICG (64.9\%). Tc99 and blue dye were used in $10.5 \%$. Only one tracer was used in 
Abstract 680 Table 1 Demographic and clinical features of the 57 included patients

\begin{tabular}{|c|c|c|c|}
\hline & Mean/median & & $\%$ \\
\hline Age (years)* & $60.2(11.9)$ & & \\
\hline BMI $\left(\mathrm{kg} / \mathrm{m}^{2}\right)^{* *}$ & $28.6(10.3)$ & & \\
\hline \multirow[t]{2}{*}{ Menopause } & & No & 28.1 \\
\hline & & Yes & 71.9 \\
\hline \multirow[t]{2}{*}{ Arterial hypertension } & & No & 57.9 \\
\hline & & Yes & 42.1 \\
\hline \multirow[t]{2}{*}{ Diabetes mellitus } & & No & 82.5 \\
\hline & & Yes & 17.5 \\
\hline \multirow[t]{2}{*}{ Previous delivery } & & No & 12.5 \\
\hline & & Yes & 87.5 \\
\hline \multirow[t]{2}{*}{ Previous Tamoxifen intake } & & No & 96.5 \\
\hline & & Yes & 3.5 \\
\hline \multirow[t]{2}{*}{ Previous cervical surgery } & & No & 93 \\
\hline & & Yes & 7 \\
\hline
\end{tabular}

Abstract 680 Table 2 Tracer detection and migration for Tc99 and ICG/Blue dye

\begin{tabular}{|c|c|c|}
\hline Tracer & $\mathrm{N}$ & $\%$ \\
\hline \multicolumn{3}{|l|}{ SPECT-TC Tc99 detection } \\
\hline No detection & 7 & 15.2 \\
\hline Pelvic unilateral & 10 & 21.7 \\
\hline Pelvic bilateral & 29 & 63 \\
\hline \multicolumn{3}{|l|}{ ICG/Blue dye migration } \\
\hline No migration & 5 & 9.2 \\
\hline Pelvic unilateral & 11 & 20.4 \\
\hline Pelvic bilateral & 38 & 70.4 \\
\hline
\end{tabular}

24.6\% (Tc99 5.3\%; ICG 17.5\%; blue dye 1.8\%). Cervix was the only injection site into submucosa and stroma.

In $89.5 \%$ of the patients, tracer migration was observed. Only 6 patients $(10.5 \%)$ had no migration. In table 2, detection and migration data of the tracers are shown.

One hundred forty-four SLNs were detected. Right side $(52.8 \%)$ was slightly more frequent than left side (47.2\%). Most SLNs were located in external iliac area $(40.5 \%)$, followed by iliac bifurcation (25.3\%), obturator fossa $(17.7 \%)$ and common iliac (13.9\%).

In three patients isolated tumor cells were detected (5.2\%) and one patient had macrometastases (1.8\%). 93\% of the patients had no pathological findings in SLNs.

Age was significantly higher in the no migration/no detection group in both Tc99 (70 vs 58.5 years; $\mathrm{p}=0.01$ ) and ICG/blue dye (72.5 vs 59.4 years; $\mathrm{p}=0.034)$. No migration/detection differences were detected for other patient's features.

Conclusion* A combined tracer technique is an effective method to detect SLNs in low-risk endometrial cancer to check lymphatic spread. Older women in our series have lower tracer migration/detection.

\section{INCIDENCE OF NODAL AND ISOLATED AORTIC METASTASES IN PATIENTS WITH SURGICALLY STAGED ENDOMETRIOID ENDOMETRIAL CANCER}

I Jaunarena*, R Ruiz, M Gorostidi, J Cespedes, D Del Valle, P Cobas, A Lekuona. Donostia Hospital, Obstetrics and Ginecology, San Sebastian, Spain

\subsection{6/ijgc-2021-ESG0.180}

Introduction/Background* Our objective was to describe the incidence of lymph node metastasis in patients with surgically staged endometrioid-type endometrial cancer in Donostia Hospital and evaluate the presence of isolated aortic metastasis. We believe, based on recent literature and our experience, that the number of lymph nodes involved in the aortic area is higher than traditionally reported.

Methodology A prospective observational study was conducted between 13 June 2014 and 31 December 2020 with 333 patients that underwent laparoscopic surgery for endometrial cancer at our institution. In all low, intermediate, high-intermediate and high ESGO/ESTRO/ESP 2020 prognostic risk cases, we performed sentinel lymph node (SLN) biopsy with dual cervical and fundal indocyanine green injection. All SLNs were processed with an ultrastaging technique. A total of 152 patients also underwent total pelvic and paraaortic lymphadenectomy.

Result(s)* The detection rates were as follows: 94\% overall for SLNs; 91.3\% overall for pelvic SLNs; 70.5\% for bilateral SLNs; $67.2 \%$ for paraaortic SLNs, $53.6 \%$ in 3 areas. A median of one aortic node and two pelvic nodes were removed. We detected positive SLNs in 56 patients $(16.8 \%$ of total cases); both pelvic and aortic SLNs were positive in 10 cases (representing 3\% of the sample; $17.8 \%$ of the total number of patients with positive nodes), while only pelvic SLNs were positive in $32(9.6 \% ; 57.1 \%)$ and only aortic SLNs were positive in $14(4.2 \% ; 25 \%)$. Sorting by myometrial infiltration, $<50 \%$ and $>=50 \%$, aortic isolated SLNs were positive in 4 and 10, representing $7.1 \%$ and $17.8 \%$ of the total with positive nodes (Fischer-Test Statistically significant), respectively.

Conclusion* In our series, the incidence of isolated aortic nodal metastasis is high compared with published reports. SLN biopsy allows a high rate of aortic detection, identifying a non-negligible percentage of isolated aortic metastases. Aortic metastases in endometrial cancer are possible and we should not give up actively looking for them. The highest rate of isolated aortic metastases occurred in patients with high-risk tumors, although there were also cases in patients with lowrisk tumors.

\section{ADVANCED ENDOMETRIAL CANCER SURGERY: WHAT REALLY IMPACTS ON SURVIVAL?}

D Tsolakidis*, E Markopoulou, S Pitis, D Zouzoulas, C Zymperdikas, M Lioupis, V Korvesi, M Topalidou, E Timotheadou, A Papanikolaou, G Grimbizis. General Hospital of Thessaloniki 'Papageorgiou', 1st Department of Obstetrics and Gynecology

\subsection{6/ijgc-2021-ESG0.181}

Introduction/Background* Endometrial cancer is the most common gynecological malignancy and its incidence is increasing steadily. More than $10 \%$ of cases will present with advanced stage disease. In these patients, the role and the time of surgery needs further clarification.

Methodology Retrospective analysis of all advanced endometrial cancer cases treated in our department from 2012 to 2018 was performed. Demographic and clinical variables were collected. Charlson comorbidity index was used to assess comorbidity. The use of neoadjuvant chemotherapy and the type of cytoreductive surgery (primary or interval debulking), surgical variables and residual disease after cytoreduction (no macroscopic disease, optimal cytoreduction $<1 \mathrm{~cm}$, residual $>1 \mathrm{~cm}$ or unresectable disease) were reported. Tumor characteristics were retracted from pathology reports. Descriptive 